\title{
Dispensing Bottle
}

National Cancer Institute

\section{Source}

National Cancer Institute. Dispensing Bottle. NCI Thesaurus. Code C43170.

A bottle that is used by the pharmacist to dispense the prescribed medication. It includes preparations for which a dropper accompanies the bottle. 